\title{
Using Tag Clouds to Explore Text Documents on Small Mobile Devices
}

\author{
Tanja Döring ${ }^{1}$, Annika Kaltenhauser ${ }^{2}$, Rainer Malaka ${ }^{1}$
}

AG Digitale Medien, TZI, Universität Bremen ${ }^{1}$

UMTL, Universität des Saarlandes ${ }^{2}$

\begin{abstract}
In this paper, we present a novel interactive tag cloud to explore large text documents on small mobile devices. While tag clouds have been popular in the World Wide Web for many years, a systematic adaptation of their properties to the requirements of small devices has yet not been addressed. With our work, we apply tag clouds to access conference proceedings on a mobile device. In a user-centered design process we derived design guidelines and evaluated a working prototype. Our results reveal that mobile tag clouds work well with a maximum of 25 tags per cloud, that they can be segmented into sub tag clouds, and that users prefer the selection of multiple tags to refine the result list of articles.
\end{abstract}

\section{Introduction}

Although tag clouds have been popular for information overview in the World Wide Web for many years (e.g., Rivadeneira et al. 2007), they so far mainly have been designed for desktop use. Despite their potentials to offer dense information overviews, besides few examples (e.g., Baldauf \& Simon 2010) no systematic approaches for their use on small devices exist yet. We address this issue and provide insights in how to adapt tag clouds for small devices. Moreover, we investigate the utility of the cloud for exploring text documents on small devices, a task that can be cumbersome having only standard search functionality. In this paper, we present design requirements for tag clouds on mobile devices, a prototype that uses an interactive segmented cloud with multiple selection for browsing proceedings on smartphones and a user study comparing the mobile tag cloud to a conventional one.

\section{Design Requirements for Mobile Tag Clouds}

Tag clouds have a number of design parameters that can be adapted to specific tasks (c.f. Rivadeneira et al. 2007, Lohmann et al. 2009, Bateman et al. 2008). To identify design 
requirements for mobile tag clouds, we conducted qualitative interviews (lasting 30-90 minutes) with 8 users (3 females, M: 37.6 years, SD: 13,9), in which we asked them to perform typical text exploration tasks with think aloud method and to assess different designs for tag clouds with varying number of tags, colours, fonts, and layouts. The tag clouds were presented on paper-based prototypes and an HTC One smartphone (4.7 inch display). In brief, our study resulted in the following design insights:

- Layout: A mobile tag cloud should use the full screen space. Users preferred a scattered and a slightly shifted alignment of tags with ragged margin to enable a better targeting of the tags. A tag size relative to term frequency was considered as useful. To have the most occurring tags well reachable, the participants preferred a circular alignment of the tags.

- Font: Selected fonts should be sans serif. Font size and distance between the tags should be sufficient to be touchable with fingers (i.e., in our case a minimal font size of $23 \mathrm{pt}$ and distance of $5 \mathrm{pt}$ ). For a better legibility, users preferred tags in different colours.

- Number of Tags: Based on the limited screen space and a sufficient font size, our study revealed to put not more than 25 tags into one tag cloud. As the tag cloud should be used for the exploration of text documents with larger amounts of tags this led to a segmented tag cloud concept consisting of multiple pages with sub tag clouds.

- Multiple Tag Selection: To allow a fine-grained exploration of large text documents the mobile tag cloud should support the selection and combination of multiple tags and generate a result list in a separate tab instead of directly linking to the results when a tag is clicked. A preview of the number of results should be shown directly.

\section{An Interactive Tag Cloud for Mobile Devices}

In order to investigate the user experience of tag clouds for large text exploration, we implemented a mobile web-based prototype based on a real data set of former Mensch und Computer (MuC) proceedings including 214 articles. To generate the cloud, we used the keywords of the articles given by the authors (with common words like articles and prepositions removed) and aligned them, according to the above-described requirements, in a font size according to term frequency beginning with the most often occurring tags in the screen centre in a circular, scattered alignment. To allow a limit of 25 tags per cloud, we realized a segmented tag cloud consisting of four cloud pages with 100 tags in total. As the tags in each cloud, they were ordered by term frequency. Users can easily browse between the tag clouds with a swipe gesture. A further novel feature of our multi-page tag cloud is multiple tag selection. When selected via tap, a tag gets highlighted (in grey with underscore), and all articles that are tagged with the corresponding keyword are added to the list of results in a different tab. To further refine the selection, more tags can be selected through all tag clouds. All selected tags are displayed on the bottom, and the list of results is adjusted to articles that contain the selected tags. Next to the cloud, the system contains views for the result list and details for each article as visible in Figure 1. 


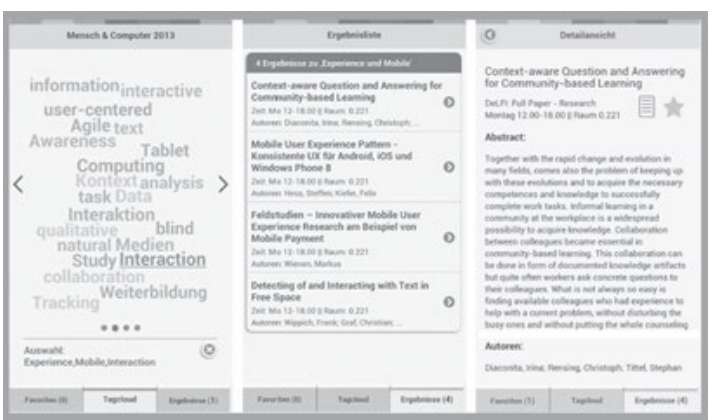

Figure 1: Left: The interactive mobile tag cloud to explore proceedings. The tag cloud allows multiple selections of tags. It is subdivided into four pages. Middle and Right: Further views of the system, list of results and detail page of selected article.

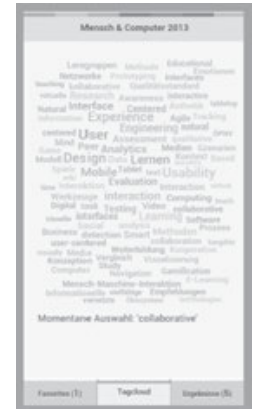

Figure 2: The conventional tag cloud realized for the comparative user study. The further screens of the system (result list and detail pages) remained the same.

\section{User Study and Results}

With our mobile tag cloud we conducted a user study in which we compared it to a conventional tag cloud as presented in Figure 2. It showed all 100 tags on one page (at the benefit of a more comprehensive overview and the cost of smaller font sizes) and, when a tag was clicked, it directly showed the corresponding result list. Pages for result list and details were the same in both prototypes. We conducted a within-group study with 8 participants, of whom 4 started with the novel cloud. In the study, we used an HTC One smartphone again and asked the users to perform 4 typical tasks. First: search for an interesting article that fits your field of interest; second: find a tag with 40 corresponding articles; third: find articles that are tagged with "media", fourth: find articles that are tagged with "usability" and "test". As we were interested in qualitative feedback we applied the think aloud method and did not measure task duration (as think aloud influences it). We additionally asked predefined questions about the system and users' preferences. Furthermore, we used the User Experience Questionnaire (UEQ) (Laugwitz et al. 2008). Our 8 users (3 female, M: 25,1 years, SD: 3,6) all had an academic background in digital media. The sessions lasted from 25 to 45 minutes. Overall, the study showed that all participants understood the concept of the multi-page tag cloud and the order by descending frequency. All changed back and forth between the cloud pages to find an interesting article in task 1 already. The users explicitly liked the multiselection feature of the cloud, which was specifically useful for task 4 , but had been used for the other tasks already. For task 2, finding a tag with at least 40 articles, between one and five taps were needed in both interface types. Here, the conventional tag cloud had the advantage that all tag sizes could be seen and compared at a glance, while in the mobile tag cloud tags were spread over several pages. Nevertheless, as the order of the tags by frequency was understood, this did not present a problem. The preview showing the number of items in the result list beneath the cloud was regarded as helpful. In task 3 users had to find articles tagged with a tag that was located on page 3 of the mobile tag cloud. Using the conventional tag cloud, 5 users found the tag fast and 3 had difficulties due to the large number of items. When using the mobile tag cloud, 2 of the participants swapped pages 
unnecessarily often, but all found the tag. In general, the users were able to achieve all tasks with both tag clouds. 5 users stated that they preferred the novel tag cloud for browsing proceedings. One preferred the other tag cloud and the remaining liked aspects in both systems. The UEQ results show that both systems were perceived as very positive. On a 7 point scale the mean values vary from 4,69 (stimulation conv. tag cloud; SD: 1,1) to 6,13 (perspicuity mobile tag cloud; SD: 0,43) with slightly better results for the mobile tag cloud in all dimensions. We evaluated the results with Wilcoxon-Mann-Whitney test. According to the test, the results between both system versions did not differ significantly, but we found a trend for a better dependability for the mobile tag cloud $(\mathrm{p}=0,082<0,1)$.

\section{Conclusion}

We developed design guidelines for tag clouds to explore large text documents on small mobile devices and built a working prototype for browsing proceedings. The mobile tag cloud was separated into sub tag clouds and allowed multiple selections of tags as filter function for refining a result list. We found that users easily understood the structure and functions of the tag cloud. Especially the multi-selection feature was regarded as very useful. We believe our tag cloud design represents a promising direction to develop further versions of tag clouds to support the work with large text documents on small devices. Future work includes a study measuring task duration and errors as well as exploring further mobilespecific tag cloud variations.

\section{Acknowledgements}

We thank the German Research Foundation (DFG) for funding this work (MA 1766/4).

\section{References}

Baldauf, M. \& Simon, R. (2010). Getting Context on the Go: Mobile Urban Tag Exploration with Ambient Tag Clouds. Proc. of the 6th Workshop on Geographic Information Retrieval GIR'10, ACM.

Bateman, S., Gutwin, C. \& Nacenta, M. (2008). Seeing things in the clouds: the effect of visual features on tag cloud selections. Proc. of HT '08, ACM, pp.193-202.

Laugwitz, B., Held, T. \& Schrepp, M. (2008). Construction and evaluation of a user experience questionnaire. Proc. of USAB 2008, LNCS 5298, pp. 63-76.

Lohmann, S., Ziegler, J. \& Tetzlaff, L. (2009). Comparison of Tag Cloud Layouts: Task-Related Performance and Visual Exploration. Proc. of INTERACT '09, Springer, pp. 392-404.

Rivadeneira, A. W., Gruen, D. M., Muller, M. J. \& Millen, D. R. (2007). Getting our head in the clouds: toward evaluation studies of tagclouds. Proc. of CHI '07, ACM, pp. 995-998.

\section{Contact}

Tanja Döring, Annika Kaltenhauser, Rainer Malaka

AG Digitale Medien, Universität Bremen / UMTL, Universität des Saarlandes

28359 Bremen, Bibliothekstr. 1 / 66123 Saarbrücken, Stuhlsatzenhausweg 3

doering@tzi.de,s9aakalt@stud.uni-saarland.de,malaka@tzi.de 